\title{
Repressing LXRs to starve cancer
}

Tumour cells exhibit unique metabolic profiles that promote rapid growth and survival. A new study has found that a small-molecule inhibitor of liver X receptors (LXRs) - which regulate the expression of key glycolytic and lipogenic enzymes involved in metabolism - is specifically toxic to cancer cells and has a favourable safety profile.

Glucose metabolism in cancer cells occurs primarily through glycolysis, even when oxygen is available; this phenomenon, known as the Warburg effect, provides cancer cells with a surplus of metabolic intermediates. Moreover, to obtain lipids, cancer cells rely more heavily on de novo lipid synthesis rather than exogenous sources. Increased expression of glycolytic and lipogenic enzymes, which underlie such

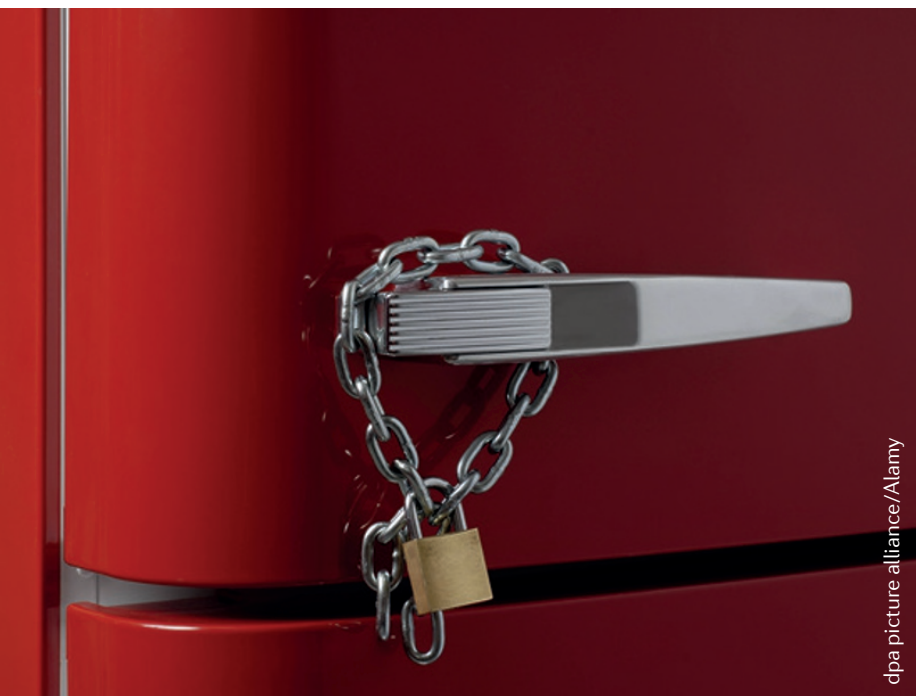

metabolic changes, is associated with cancer aggressiveness and drug resistance. Efforts to therapeutically target these enzymes have been hampered, however, by adverse effects such as severe weight loss and high toxicity.

In the current study, Flaveny et al. postulated that targeting LXRs might enable a more nuanced approach to hone in on cancer cell metabolism. When activated by endogenous agonists, these nuclear receptors directly bind to and regulate the expression of LXR-responsive genes. Conversely, when unliganded, the receptors recruit co-repressors, leading to the suppression of target gene expression.

The researchers focused on the small molecule SR9243, an LXR inverse agonist that binds to unliganded LXRs and promotes co-repressor recruitment, thereby 'switching off' constitutive LXR activity. In a range of cancer cell lines - including prostate, colorectal and lung cancer - the compound potently reduced cancer cell viability and replication, causing cell death within $12 \mathrm{~h}$ of treatment. By contrast, non-malignant cells expressing similar levels of LXRs to cancer cells were unaffected. SR9243 also sensitized cancer cells to treatment with $5^{\prime}$-fluorouracil or cisplatin, suggesting that it might be useful for enhancing chemotherapy.

Consistent with the hypothesis that SR9243 mediates its anticancer effect in part through inhibition of the Warburg effect, Flaveny et al. found that treatment significantly reduced the expression of glycolytic enzymes, including glucokinase 1 and phosphofructokinases, in malignant cells but not in normal cells. Intracellular levels of glycolytic metabolites such as pyruvate were also significantly attenuated by treatment with the drug in cancer cells.

Following a similar pattern, SR9243 significantly repressed lipogenesis enzyme genes that are under the regulation of LXRs, and it reduced intracellular levels of lipogenesis end products, such as palmitate, in cancer cell lines but not in normal cells.

Last, the researchers confirmed their in vitro findings in mice, showing compound-induced inhibition of glycolysis and lipogenesis as well as increased apoptotic cancer cell death in colon cancer tumours engrafted in mice. Importantly, SR9243 treatment reduced tumour growth without causing weight loss. Treatment also increased the tumour-specific expression of tumour necrosis factor, a form of immune activation that the authors suggested could represent tumour 'unmasking' to the immune system.

Overall, the specificity of the effects of SR9243 for tumour cells across a range of cancers and the lack of notable adverse effects highlight inverse agonists of LXRs as potential anticancer treatments.

Katie Kingwell

ORIGINAL RESEARCH PAPER Flaveny, C. A. et al. Broad anti-tumor activity of a small molecule that selectively targets the Warburg effect and lipogenesis. Cancer Cell 28, 42-56 (2015) 